\title{
Acupuncture techniques for COPD: a systematic review
}

\author{
Carles Fernández-Jané ${ }^{1,2^{*}}$, Jordi Vilaró ${ }^{1,2}$, Yutong Fei ${ }^{3}$, Congcong Wang ${ }^{3}$, Jianping Liu ${ }^{3}, \mathrm{Na} \mathrm{Huang}^{3}$, Ruyu Xia ${ }^{3}$, \\ Xia Tian ${ }^{3}$, Ruixue $\mathrm{Hu}^{3}$, Lingzi Wen ${ }^{3}$, Mingkun $\mathrm{Yu}^{3}$, Natàlia Gómara-Toldrà ${ }^{1,4}$, Mireia Solà-Madurell ${ }^{5}$ \\ and Mercè Sitjà-Rabert ${ }^{1,2}$
}

\begin{abstract}
Background: This is the second part of a large spectrum systematic review which aims to identify and assess the evidence for the efficacy of non-pharmacological acupuncture techniques in the treatment of chronic obstructive pulmonary disease (COPD). The results of all techniques except for filiform needle are described in this publication.

Methods: Eleven different databases were screened for randomised controlled trials up to June 2019. Authors in pairs extracted the data and assessed the risk of bias independently. RevMan 5.3 software was used for the metaanalysis.
\end{abstract}

Results: Thirty-three trials met the inclusion criteria, which involved the follow techniques: AcuTENS (7 trials), moxibustion (11 trials), acupressure (7 trials), ear acupuncture ( 6 trials), acupressure and ear acupuncture combined ( 1 trial) and cupping ( 1 trial). Due to the great heterogeneity, only 7 meta-analysis could be performed (AcuTENS vs sham on quality of life and exercise capacity, acupressure vs no acupressure on quality of life and anxiety and ear acupuncture vs sham on $\mathrm{FEV}_{1}$ and $F E V_{1} / F V C$ ) with only acupressure showing statistical differences for quality of life (SMD: -0.63 95\%Cl: $-0.88,-0.39 \mathrm{I}^{2}=0 \%$ ) and anxiety (HAM-A scale MD:-4.83 95\%Cl: $-5.71,-3.94 \mathrm{I}^{2}=0 \%$ ).

Conclusions: Overall, strong evidence in favour of any technique was not found. Acupressure could be beneficial for dyspnoea, quality of life and anxiety, but this is based on low quality trials.

Further large well-designed randomised control trials are needed to elucidate the possible role of acupuncture techniques in the treatment of COPD.

Trial registration: PROSPERO (identifier: CRD42014015074).

Keywords: COPD, Acupuncture therapy, Dyspnoea, Quality of life, Systematic review, Meta-analysis

\section{Introduction}

Chronic obstructive pulmonary disease (COPD) is one of the most prevalent lung diseases, with 251 million cases globally in 2016, and is the 4th cause of death worldwide, with more than 3.2 million instances in 2015 [1]. These numbers are expected to increase [2].

\footnotetext{
* Correspondence: carlesfj@blanquerna.url.edu

${ }^{1}$ School of Health Science Blanquerna, Ramon Llull University, Barcelona, Spain

${ }^{2}$ Global Research on Wellbeing (GRoW) Research Group, Ramon Llull University, Padilla 326-332, 08025 Barcelona, Spain

Full list of author information is available at the end of the article
}

COPD is characterised by a chronic and irreversible airflow obstruction caused by an inflammation in the airways and lung parenchyma which leads to structural abnormalities in the airways. These alterations specially affect force expiratory volume in the first second $\left(\mathrm{FEV}_{1}\right)$ compared to force vital capacity (FVC) [3]. The main symptoms of this disease are progressive dyspnoea, chronic cough, sputum production and recurrent respiratory infections. Those symptoms get worse as the disease evolves, with many effects on exercise capacity and quality of life [4].

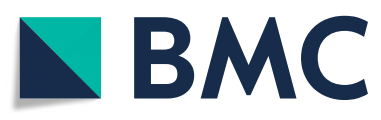

(c) The Author(s). 2020 Open Access This article is licensed under a Creative Commons Attribution 4.0 International License, which permits use, sharing, adaptation, distribution and reproduction in any medium or format, as long as you give appropriate credit to the original author(s) and the source, provide a link to the Creative Commons licence, and indicate if changes were made. The images or other third party material in this article are included in the article's Creative Commons licence, unless indicated otherwise in a credit line to the material. If material is not included in the article's Creative Commons licence and your intended use is not permitted by statutory regulation or exceeds the permitted use, you will need to obtain permission directly from the copyright holder. To view a copy of this licence, visit http://creativecommons.org/licenses/by/4.0/ The Creative Commons Public Domain Dedication waiver (http://creativecommons.org/publicdomain/zero/1.0/) applies to the data made available in this article, unless otherwise stated in a credit line to the data. 
The usual treatment in COPD targets its main symptoms. Pharmacological treatment includes the use of corticosteroids and bronchodilators to reduce airway inflammation and obstruction, and non-pharmacological treatment such as pulmonary rehabilitation are used to improve perceived dyspnoea, exercise capacity and quality of life [3].

Acupuncture derives from Traditional Chinese Medicine, which uses different techniques to stimulate specific areas of the body surface, or acupuncture points, to restore health. Even though inserting needles is the bestknown acupuncture technique (filiform needle acupuncture), there are several others, including heat stimulation (moxibustion), electricity (electroacupuncture or acupoint transcutaneous electrical nerve stimulation (AcuTENS)), and digital pressure (acupressure). These techniques have been traditionally used to treat all kinds of health problems including respiratory diseases like COPD, however there is little evidence about the effectiveness of those techniques and no previous review has studied different acupuncture techniques individually.

The aim of this review is to identify and separately evaluate the efficacy of non-pharmacological acupuncture techniques, excepting via filiform needle. These techniques include moxibustion (except when performed alongside use of an acupuncture needle), electroacupuncture (when not delivered using an acupuncture needle) AcuTENS, acupressure and ear acupuncture, and cupping therapy among others.

\section{Methods}

\section{Protocol and registration}

We followed the recommendations of the Cochrane Handbook for Systematic Reviews of Interventions [5] for this review and the Preferred Reporting Items for Systematic Reviews and Meta-Analyses (PRISMA) statement [6]. The protocol was previously registered at PROSPERO (CRD42014015074) and is available on: http://www.crd.york.ac.uk/PROSPERO/display_record. asp?ID=CRD42014015074).

\section{Eligibility criteria}

We included randomised controlled trials or quasirandomised trials and crossover trials, meeting all the following criteria: [1] performed in COPD patients with different grades of obstruction (GOLD A to D) in exacerbation or stable periods [2]; assessing nonpharmacological modalities of acupuncture (filiform needle, electroacupuncture, acupressure, moxibustion, ear acupuncture, etc.) compared with a control group (sham acupuncture or no acupuncture), in addition to usual care (medication, physiotherapy, pulmonary rehabilitation, etc.); and [3] reporting at least one of the following outcomes: dyspnoea, quality of life, adverse effects, exercise capacity, lung function or anxiety and depression.

Exclusion criteria were: [1] if acupuncture was compared with a different acupuncture technique or a therapy not used in usual care; and [2] randomised cluster studies.

No language restriction was applied.

Due to the large number of acupuncture techniques found, we decided to exclude those that were mainly used only in China and to focus on those best known and practiced elsewhere in the world. Trials involving the techniques are listed in the results section, but were not analysed. This exclusion criteria was not applied in our original protocol.

\section{Information sources}

An electronic search was performed up to June 2019. The databases included were the Cochrane Central Register of Controlled Trials (CENTRAL), Medline, Embase, CINAHL, AMED (Ovid), PEDro, PsycINFO, CNKI, VIP, Wanfang and Sino-Med. the bibliographies of selected articles were also consulted in search of additional studies not detected in the initial searches. Manual reviews were also performed on international respiratory diseases conferences (European Respiratory Society and American Association for Respiratory Care) from 2010 to 2017.

\section{Search}

We conducted a comprehensive search using the following key words and their variations: "acupuncture", "moxibustion", "acupressure", "electroacupuncture", "AcuTENS", "ear acupuncture", "cupping", "COPD", "randomised control trial". The search strategy was adjusted for each database (see Supplementary material 1).

\section{Study selection}

The reviewers (CF), MSR, JV, WC, HN, XRY, TX, HRX MS, NGT) worked in pairs and independently identified the articles that met the inclusion criteria, first through title and abstract and afterwards through full text paper.

\section{Data collection process}

Reviewers (CFJ, MSR, JV, WC, HN, XRY, TX, HRX MS, NGT), both in pairs and independently, extracted data using a standardised data extraction form. A pilot test was performed prior to data extraction to check the suitability of the form, as well as its understanding by the reviewers. A third author was consulted in the case of discrepancies. Lack of data or inconsistent data were managed by contacting trial authors; if this was not possible the data was not included in the meta-analysis. 


\section{Risk of bias in individual studies}

The Cochrane Risk of Bias Assessment Tool [7] was used to assess the risk of bias in the papers. Due to the nature of acupuncture techniques, the Cochrane risk of bias tool was modified to add "blinding of outcome assessment". "Blinding of personnel" was removed because a person providing acupuncture treatment cannot be blinded.

\section{Summary measures}

Continuous outcomes were expressed as mean difference (MD) with 95\% confidence interval (CI) or standardised mean difference (Std. MD) when different scales were used. For trials with different arms using acupuncture, the results were combined before meta-analysis using the Cochrane Handbook [8].

\section{Synthesis of results}

The heterogeneity of the studies was evaluated using the $\mathrm{I}^{2}$ statistic. Post-treatment data from each group or post treatment differences between groups were used for the meta-analysis. When this was not reported or large baseline differences were found between the groups, the difference from baseline data from each group were used. The results were combined in a meta-analysis using RevMan 5.3 software and applying a fixed effects model to summarise the results when heterogeneity was not relevant $\left(\mathrm{I}^{2}<30 \%\right)$. Otherwise, a random effects model was used. If $\mathrm{I}^{2}$ value was over $70 \%$, a narrative synthesis of the available data was performed.

\section{Additional analyses}

Since studies included patients with different conditions (stable and exacerbation) and this could lead to heterogeneity in our results, we decided to separate them into two subgroups in all meta-analyses. The results are therefore presented separately when heterogeneity was too big $\left(\mathrm{I}^{2}<70 \%\right)$ between subgroups or in one of the subgroups.

\section{Results}

\section{Study selection}

To identify potentially eligible studies, reviewers in pairs independently screened all 5030 unduplicated titles and abstracts retrieved, and the full text of 163 articles was obtained for decisions about final inclusion. Forty-eight articles were excluded for the reasons shown in Fig. 1. As mentioned in the methods section, several acupuncture techniques used only in China were not included in the analysis: catgut implant (17 studies), tree-edge needle (1study), thumb-tack needle (1 study), thick needle ( 2 studies), acupoint incision (1 study), wet cupping (3 studies) or floating needle (1 study) and intradermal needle (1 study). Sixty-two studies (36 publications) were included and analysed in the review.
In this publication we included the results from 35 trials (36 publications) which used all other techniques except filiform needle: AcuTENS (7 trials) [9-15], moxibustion (13 trials) [8, 16-27], acupressure (7 trials from 8 publications) [28-35], ear acupuncture (6 trials) [36-41], acupressure combined with ear acupuncture (1 trial) [42] and cupping technique (1 trial) [43] (one trial with multiple arms was included in the filiform needle group and the ear acupuncture group) (Fig. 1).

\section{Study characteristics}

The details of all trials included, classified for intervention, are summarised in Table 1.

\section{Design}

All trials were classified as randomised control trials since they all reported that groups were generated randomly, however, 14 trials did not provide sufficient information about the sequence generation process. Only one trial used a cross-over design [30].

\section{Participants}

The mean age of participants ranged from 52 to 78 years and was similar across all interventions. From the 34 included trials, severity was not reported in 29 . Most trials included mild to severe participants. After reading each paper carefully, 29 trials were classified as treating stable patients $[8-12,14-27,29-34,37-40]$ and 6 trials were classified as treating exacerbated patients [12, 27, 35, 40-42].

\section{Interventions and comparisons}

AcuTENS Seven trials using AcuTENS were included. All trials used a similar protocol that consisted of using a stimulation pulse of between 2 and $4 \mathrm{~Hz}$ with a wave width of 200 microseconds and a stimulation time of 40-45 min. Five out of seven papers used a single point treatment, with four papers using Ding Chuan (EX-B1) $[9,11,12,15]$ and one using BL 13 [14]. Only two papers used a combination of several points $[10,13]$. The main differences between protocols were seen in the treatment regime, with two papers using a single session intervention $[9,12]$ whereas in the other five papers stimulation was used from 10 to 20 sessions and a frequency between seven and four sessions a week.

All seven trials were sham-controlled using an AcuTENS device with no electric output.

Moxibustion The thirteen studies included used multiple moxibustion techniques, including moxa stick $[16,18,20,22,23,26]$, heat sensitivity moxibustion [23, 24, 27], cone moxibustion [8, 17], moxibustion boxes [25] and ginger moxibustion [19, 21]. There were a number of acupuncture points used, ranging 


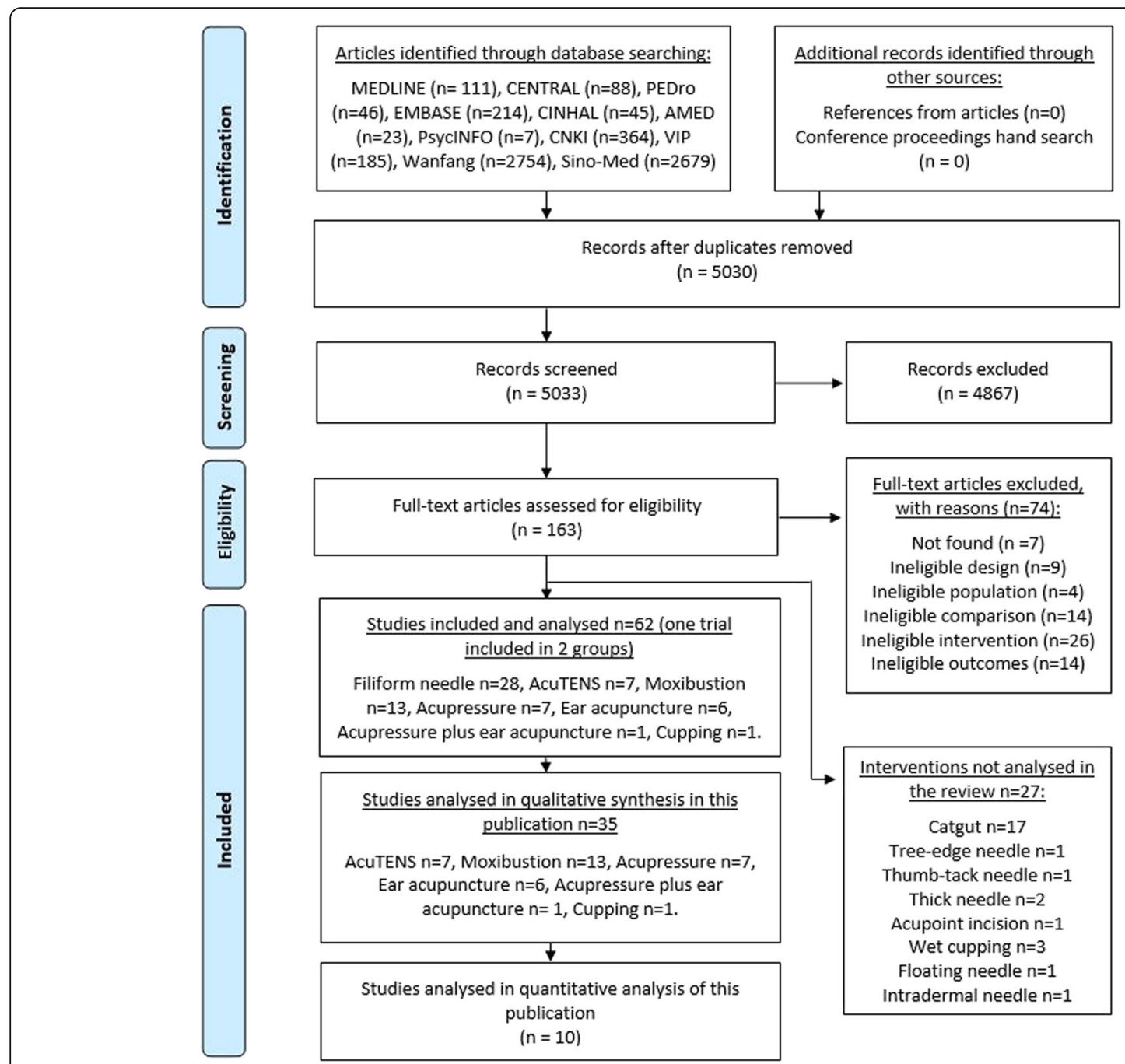

Fig. 1 Flow diagram

from 1 to 12 , but most of the trials used from 3 to 7 points [8, 18-20, 23]. Most common acupuncture points used were.

Bl13 (7 trials, 53\%), GV14, BL20 and ST36 (5 trials, $28 \%)$. Treatment regimens were also different, with two studies using from 3 to 6 treatment courses of 10-14 consecutive days during a year $[16,18]$, three studies using daily treatments for a period of 2 to 4 weeks [19, 20,23], two using from 2 to 5 treatments a week over a month $[8,21]$, two trials using 5 treatments a week over 3 to 4 months $[24,25]$, one using nine treatments every 10 days over 3 months [17] and one trial using one treatment per month [22]. In all thirteen papers, moxibustion was added to the usual treatment and compared with the usual treatment alone.

Acupressure Out of the 7 included trials using acupressure, 5 trials included used $5-7$ points $26-28,[32,34]$ while 2 trials used 3 points [33, 35]. Most common used point were GV14 (4 trials, 57\%) and BL13 (3 trials, 43\%). Most trials used rubbing and pressing stimulation (1-3 min per point), in 5 to 15 min sessions but differed in the acupoints used, and in the session regime, which ranged from at least one treatment a day to five treatments a week over 4-24 weeks [29-31]. One study differed clearly from the others in using two points of stimulation plus the ear acupuncture point Shenmen in a daily treatment regime for 10 days [28].

Acupressure was compared with sham acupressure in two studies (three publications) using the stimulation of non-specific points [29-31]. Acupressure plus the usual treatment was compared with the usual treatment alone in five trials [28, 32-35].

Ear acupuncture The six studies included used from four to seven ear acupuncture points. Most common 


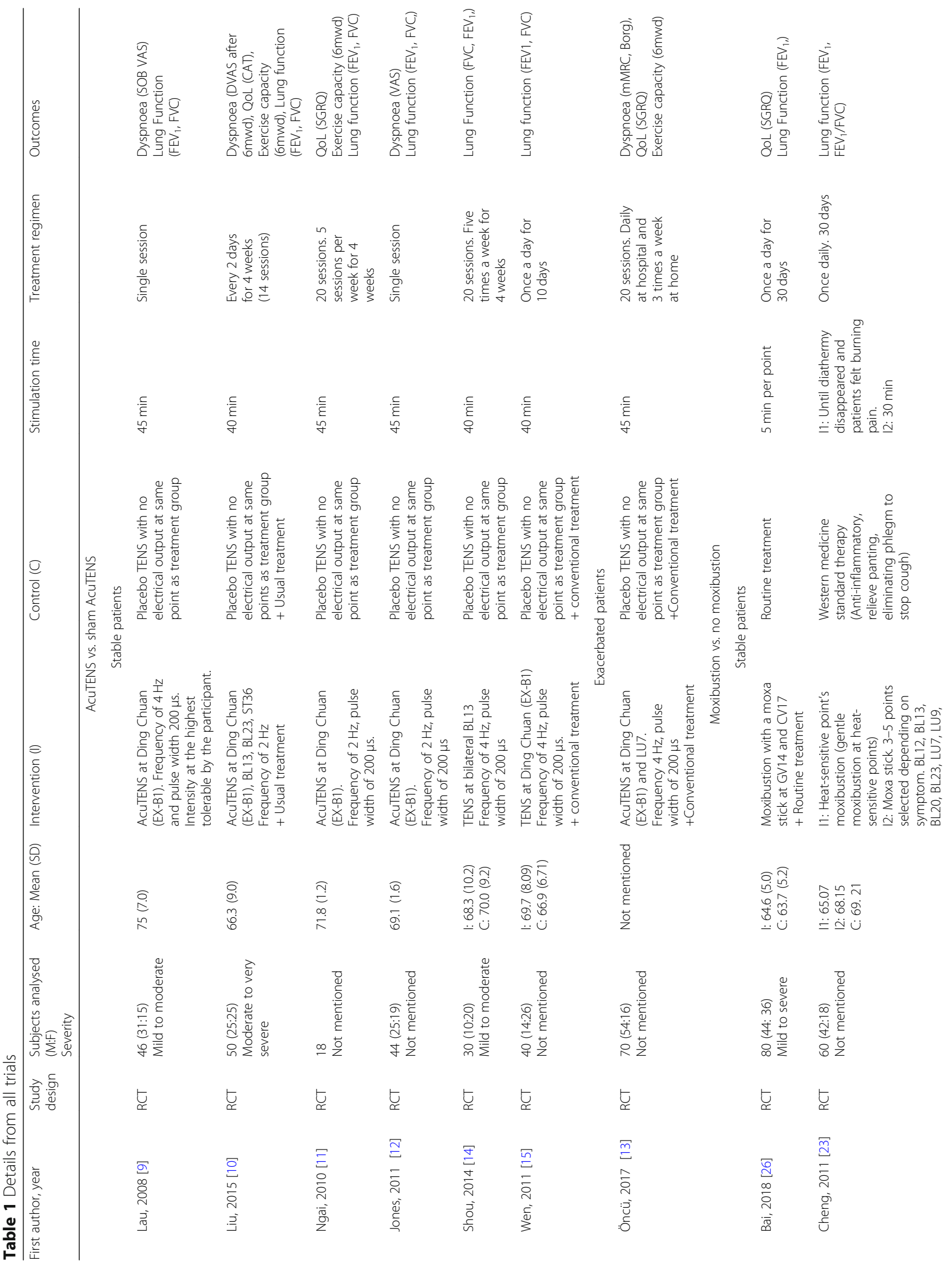




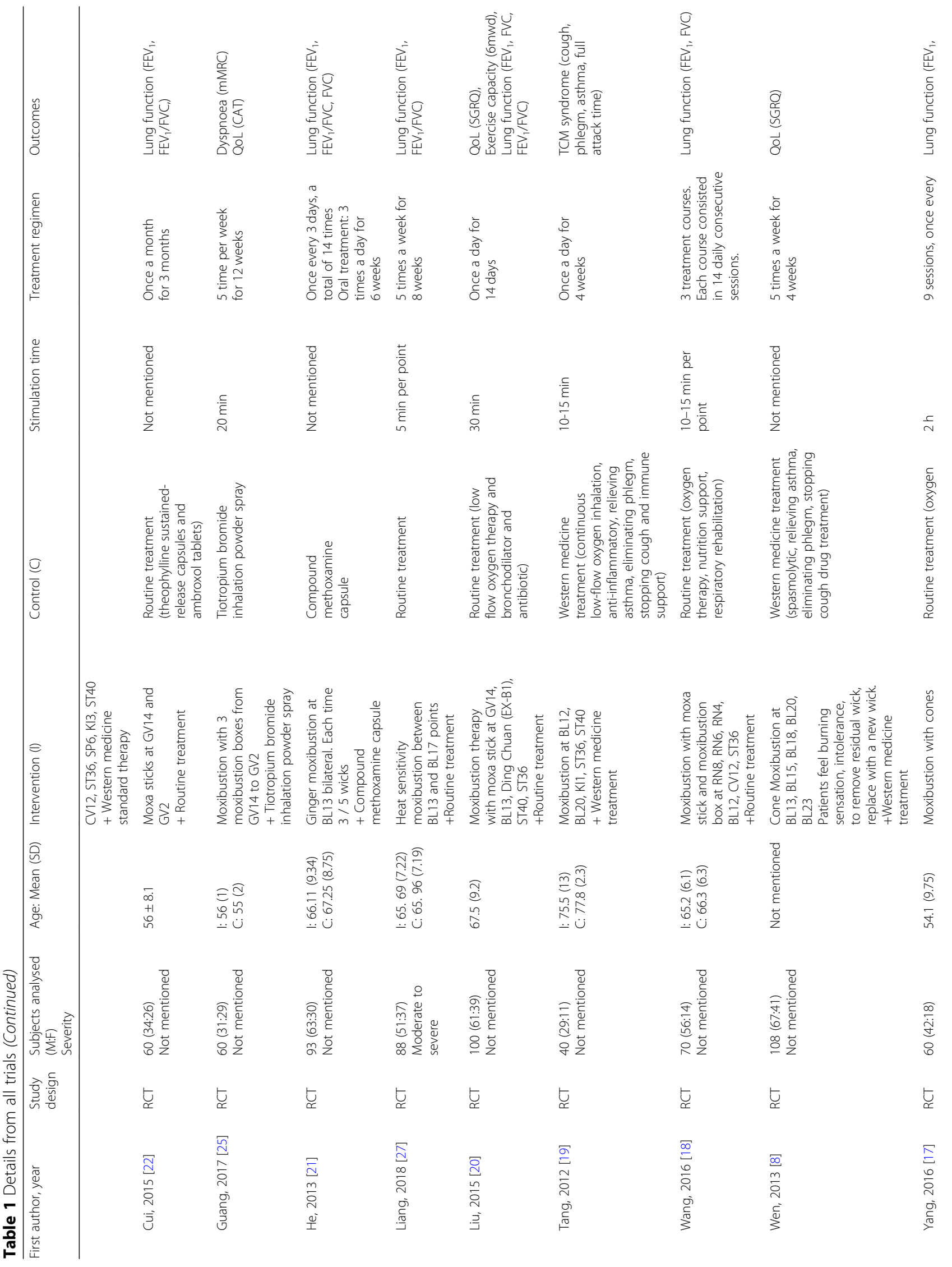




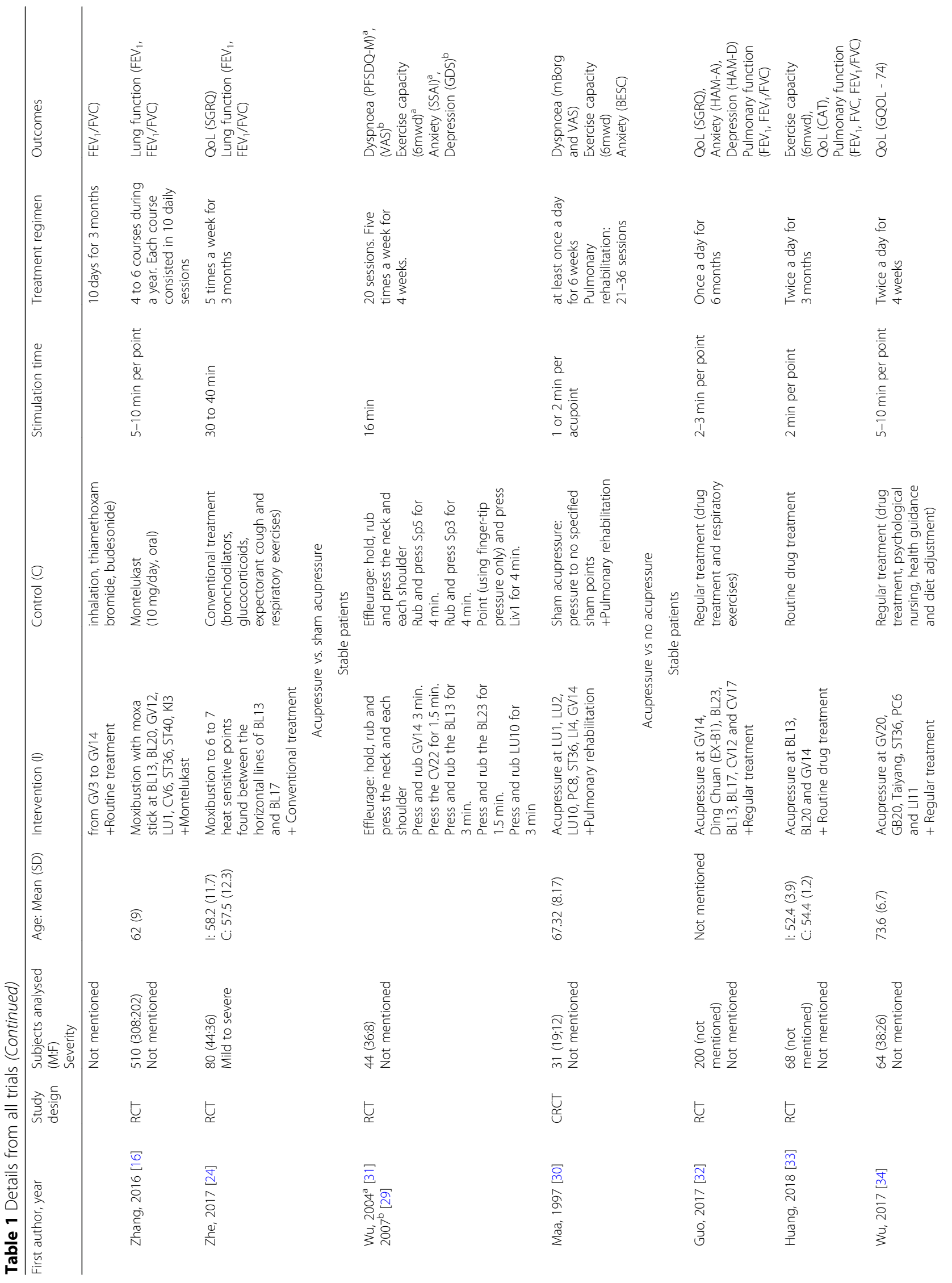




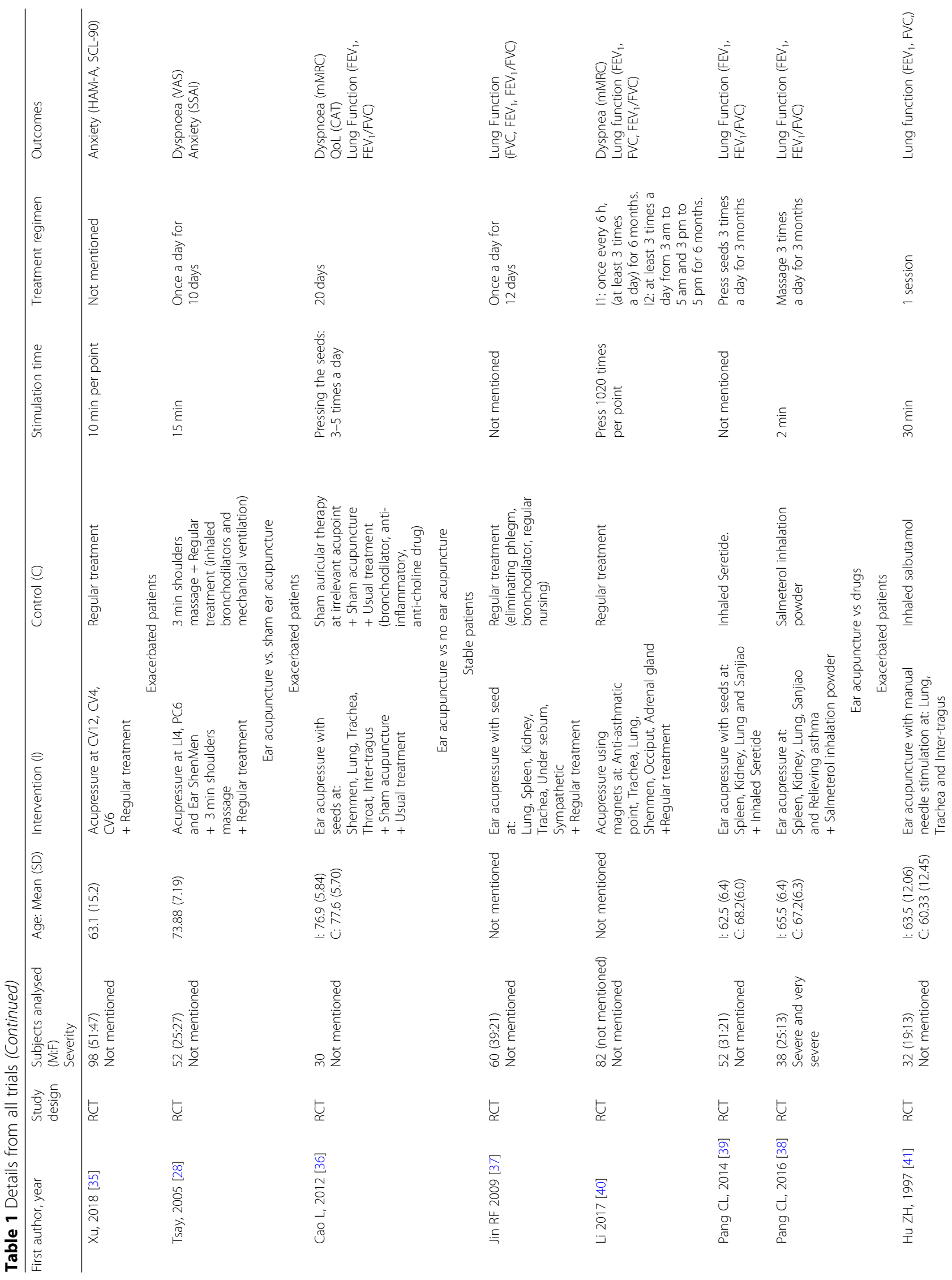




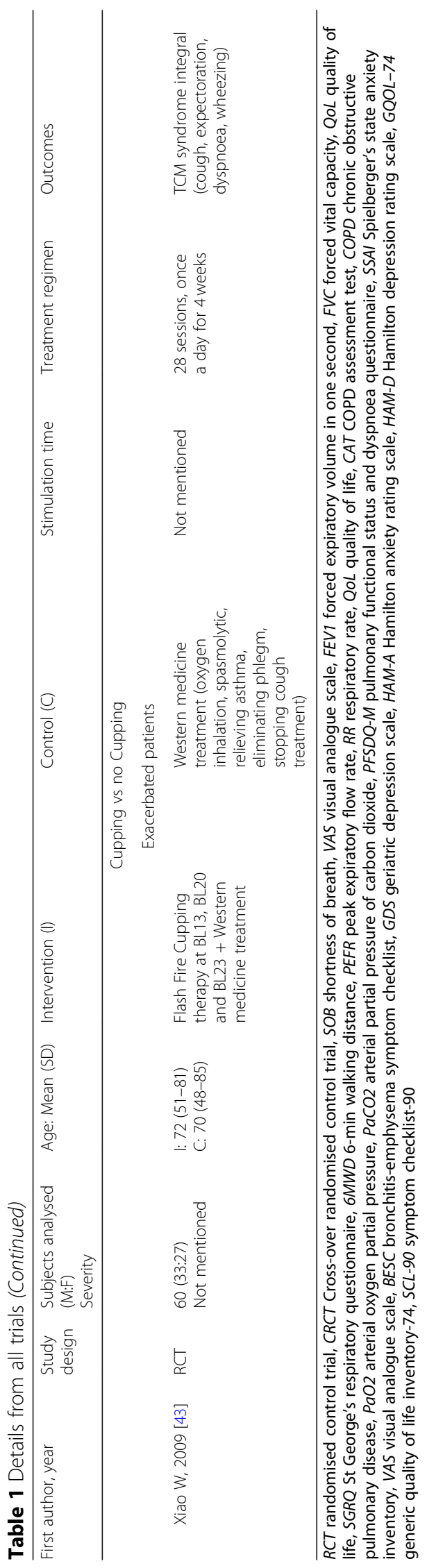


points were Lung (6 trials, 100\%) and Trachea (4 trials, $66 \%)$. Ear acupuncture was performed using seed stimulation [36-38], acupressure stimulation [39], stimulation with magnets [40] or filiform needle stimulation [41]. In four trials [36-39], the intervention duration ranged from 12 days to 6 months and only one trial used a single session treatment [41].

Sham control was used in only one trial using irrelevant ear points [36]. Four trials compared the usual treatment plus ear acupuncture with the usual treatment alone [37-40]. One compared ear acupuncture with inhaled salbutamol [41].

Acupressure plus ear acupuncture One trial combined acupressure plus ear acupuncture added to usual treatment compared with usual treatment alone [42].

Cupping One trial studied the effect of cupping plus usual treatment compared with usual treatment alone [43]. In this trial fire cupping with flash stimulation at BL13, BL20 and BL23 was used for 28 sessions for 4 weeks.

\section{Risk of bias within studies}

An assessment of the risk of bias for the included trials is summarised in Table 2.

For AcuTENS trials, seven studies (100\%) had a low risk of bias in "random sequence generation" and six (86\%) in the "blinding of participants" (the other trial was considered unclear), however the most critical items were "allocation concealment" and "blinding of outcome assessment" with five $(71 \%)$ and three $(43 \%)$ trials classified as unclear due the lack of reporting. "Selective outcome reporting" was mainly classified as unclear (6 trials) due the fact that we could not find trials protocols.

We found an important lack of reporting in all trials and items for moxibustion, meaning that there was an unclear risk of bias for this technique.

Reporting was also poor for acupressure, and therefore the risk of bias was considered unclear. Low risk of bias was considered in only four trials (57\%) for "random sequence generation", none ( $0 \%)$ for "allocation concealment", two trials (28\%) for "blinding of participants" and one (14\%) for "blinding of outcome assessment".

Only one trial (16\%) reported enough information for ear acupuncture to assess the risk of bias, which was classified low for "random sequence generation", "allocation concealment", "blinding of participants" and "blinding of outcome assessment".

Regarding the trial combining acupressure and ear acupuncture, only the risk of bias for "random sequence generation" was classified as low.

The only trial included for cupping therapy, had an unclear risk of bias in all items except "incomplete outcome data" which was classified as having a low risk of bias.

\section{Synthesis of results \\ ACUTENS}

\section{AcuTENS vs. sham AcuTENS Dyspnoea}

Four trials assessed dyspnoea, comparing AcuTENS vs sham AcuTENS, three included stable patients $[9,10$, 12] and one included exacerbated patients [13]. The Dyspnoea Visual Analogue Scale (DVAS) was used in three trials $[9,10,12]$, and the other one used the Borg Scale [13]. A meta-analysis could not be performed due to the high heterogeneity $\left(\mathrm{I}^{2}=96 \%\right)$. In the three trials with stable participants, two showed an improvement in dyspnoea in a single session treatment $[9,12]$, and the other showed no difference between groups in a 4 week treatment [10]. The trial with exacerbated participants [13] did not show any effect compared with the sham intervention (Fig. 2a).

\section{Quality of life}

Three trials assessed QoL, two including stable patients $[10,11]$ and one with exacerbated patients [13]. QoL was assessed with the St Gorge's Respiratory Questionnaire (SGRQ) in 2 trials $[11,13]$ and the COPD Assessment Test (CAT) in the other one [10]. Metaanalysis of all three trials (128 participants) did not show statistical differences between real and sham AcuTENS (Std. MD: -0.35 95\%CI: $-0.70,0.00 \mathrm{I}^{2}=0 \%$ ) (Fig. 2b).

\section{Exercise capacity}

Three trials assessed exercise capacity, two with stable patients $[10,11]$ and one including exacerbated patients [13]. All trials used the six minutes walking distance test (6MWD). Meta-analysis of the three trials (128 participants) did not show a statistical improvement between AcuTENS and Sham (6MWD MD: 6.59 95\%CI: -2.00 , $15.19 \mathrm{I}^{2}=0 \%$ ) (Fig. 2c).

\section{Lung function}

Lung function ( $\mathrm{FEV}_{1}$ and $\left.\mathrm{FVC}\right)$ was assessed in seven trials, six including stable patients [9-12, 14, 15] and one with exacerbated participants [13]. Meta-analysis was not possible for $\mathrm{FEV}_{1}$ due heterogeneity $\left(\mathrm{I}^{2}=73 \%\right)$, even in the subgroup analysis (stable subgroup $I^{2}=71 \%$ ). Of the seven trials, two $[9,10]$ showed statistic benefit for AcuTENS and five other trials indicated no difference between groups [11-15] (Fig. 2d). Meta-analysis of the seven trials [9-15] (288 participants) for FVC showed no benefit for the AcuTENS group (Std. MD: 0.12 95\%CI: - 0.16, $0.39 I^{2}=25 \%$ ) (Fig. 2e).

\section{ADVERSE EVENTS}

Only two trials attempted to report adverse events, Shou [14] reported that the technique was safe and Ngai [12] reported no associated adverse effects.

\section{MOXIBUSTION \\ Moxibustion vs. no moxibustion}

Dyspnoea Only one trial assessed dyspnoea [25]. In this trial authors reported a greater reduction on the mMRC 
Table 2 Detailed risk of bias of each trial

\begin{tabular}{|c|c|c|c|c|c|c|c|}
\hline & $\begin{array}{l}\text { Random sequence } \\
\text { generation }\end{array}$ & $\begin{array}{l}\text { Allocation } \\
\text { concealment }\end{array}$ & $\begin{array}{l}\text { Blinding of } \\
\text { participants }\end{array}$ & $\begin{array}{l}\text { Blinding of outcome } \\
\text { assessment }\end{array}$ & $\begin{array}{l}\text { Incomplete } \\
\text { outcome data }\end{array}$ & $\begin{array}{l}\text { Selective outcome } \\
\text { reporting }\end{array}$ & $\begin{array}{l}\text { Other sources } \\
\text { of bias }\end{array}$ \\
\hline \multicolumn{8}{|c|}{ AcuTENS } \\
\hline Lau, 2008 [9] & Low & Low & Low & Low & Low & Unclear & Low \\
\hline Liu X, 2015 [10] & Low & Low & Low & Low & Low & Unclear & Low \\
\hline Ngai, 2010 [11] & Low & Unclear & Low & Low & Low & Unclear & Low \\
\hline Jones, 2011 [12] & Low & Unclear & Low & Low & Unclear & High & Unclear \\
\hline Öncü, 2017 [13] & Low & Unclear & Low & Unclear & Unclear & Unclear & Unclear \\
\hline Shou, 2014 [14] & Low & Unclear & Unclear & Unclear & Low & Unclear & Unclear \\
\hline Wen Q 2011 [15] & Low & Unclear & Low & Unclear & Low & Unclear & Unclear \\
\hline \multicolumn{8}{|c|}{ Moxibustion } \\
\hline Bai 2018 [26] & Unclear & Unclear & High & Unclear & Unclear & Unclear & Unclear \\
\hline Cheng AP, 2011 [23] & Unclear & Unclear & Unclear & Unclear & Unclear & Low & Unclear \\
\hline Cui XX, 2015 [22] & Low & Unclear & Unclear & Unclear & Unclear & Low & Unclear \\
\hline Guang, 2017 [25] & Low & Unclear & High & Unclear & Unclear & Unclear & Unclear \\
\hline He F, 2013 [21] & Unclear & Unclear & High & High & Unclear & Low & Low \\
\hline Liang, 2018 [27] & Low & Unclear & High & Unclear & Unclear & Unclear & Unclear \\
\hline Liu SR, 2015 [20] & Low & Unclear & Unclear & Unclear & Unclear & Low & Unclear \\
\hline Tang J 2012 [19] & Unclear & Unclear & Unclear & Unclear & Unclear & Low & Unclear \\
\hline Wang WH, 2016 [18] & Unclear & Unclear & Unclear & Unclear & Low & Unclear & Unclear \\
\hline Wen X, 2013 [8] & Low & Unclear & Unclear & Unclear & Unclear & Low & Unclear \\
\hline Yang XQ, 2016 [17] & Low & Unclear & Unclear & Unclear & Unclear & Low & Unclear \\
\hline Zhang QY, 2016 [16] & Low & Unclear & Unclear & Unclear & Unclear & Low & Unclear \\
\hline Zhe, 2017 [24] & Low & Unclear & High & Unclear & Unclear & Unclear & Unclear \\
\hline \multicolumn{8}{|c|}{ Acupressure } \\
\hline Guo 2017 [32] & Low & Unclear & High & Unclear & Unclear & Unclear & Unclear \\
\hline Huang 2018 [33] & Low & Unclear & High & Unclear & Unclear & Unclear & Unclear \\
\hline Maa, 1997 [30] & Low & Unclear & Low & Unclear & Low & Unclear & Low \\
\hline Tsay, 2005 [28] & Unclear & Unclear & Low & Low & Low & Unclear & Unclear \\
\hline Wu, 2004 [31], 2007 [29] & Unclear & Unclear & Unclear & Unclear & Unclear & Unclear & Unclear \\
\hline Wu, 2017 [34] & Low & Unclear & High & Unclear & Unclear & Unclear & Unclear \\
\hline Xu 2018 [35] & Unclear & Unclear & High & Unclear & Unclear & Unclear & Unclear \\
\hline \multicolumn{8}{|c|}{ Ear acupuncture } \\
\hline Cao L, 2012 [36] & Low & Low & Low & Low & Low & Unclear & Unclear \\
\hline Hu ZH, 1997 [41] & Unclear & Unclear & Unclear & Unclear & Low & Unclear & Unclear \\
\hline Jin RF, 2009 [37] & Unclear & Unclear & Unclear & Unclear & Low & Low & Unclear \\
\hline Li, 2017 [40] & Unclear & Unclear & High & Unclear & Low & Unclear & Unclear \\
\hline Pang CL, 2014 [39] & Unclear & Unclear & Unclear & Unclear & Low & Unclear & Unclear \\
\hline Pang CL, 2016 [38] & Unclear & Unclear & High & Unclear & Low & Low & Unclear \\
\hline \multicolumn{8}{|c|}{ Acupressure plus ear acupuncture } \\
\hline Rao, 2017 [42] & Low & Unclear & High & Unclear & Unclear & Unclear & Unclear \\
\hline \multicolumn{8}{|c|}{ Cupping } \\
\hline Xiao W, 2009 [43] & Unclear & Unclear & High & Unclear & Low & Unclear & Unclear \\
\hline
\end{tabular}


Figure 2a. Effect on dyspnoea

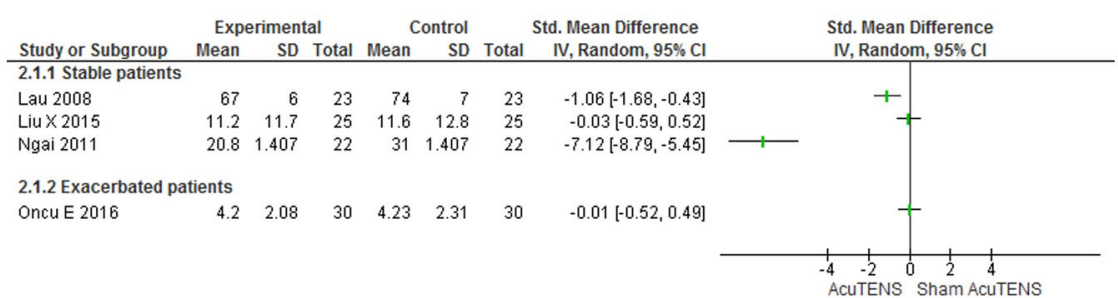

Figure $2 b$. Effect on quality of life

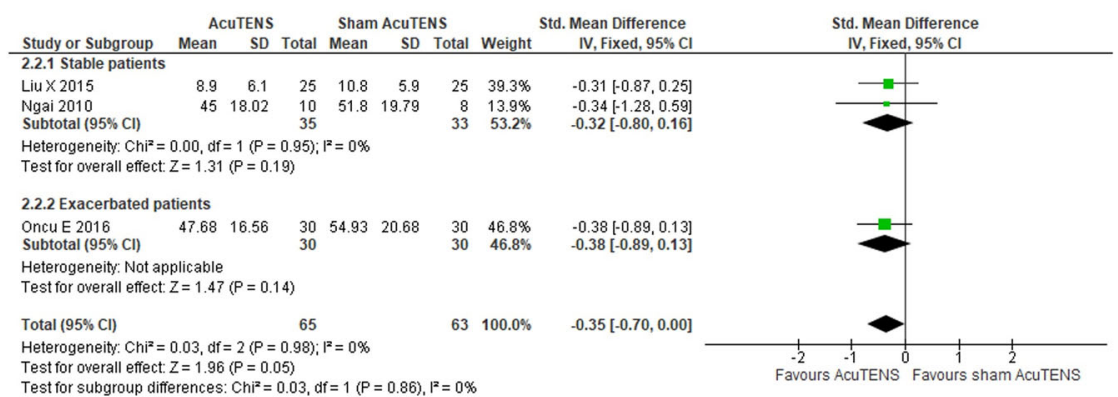

Test for subgroup differences: $\mathrm{Chi}^{2}=0.03, \mathrm{df}=1(P=0.86), \mathrm{I}^{2}=0 \%$

Figure 2c. Effect on exercise capacity (6MWD)

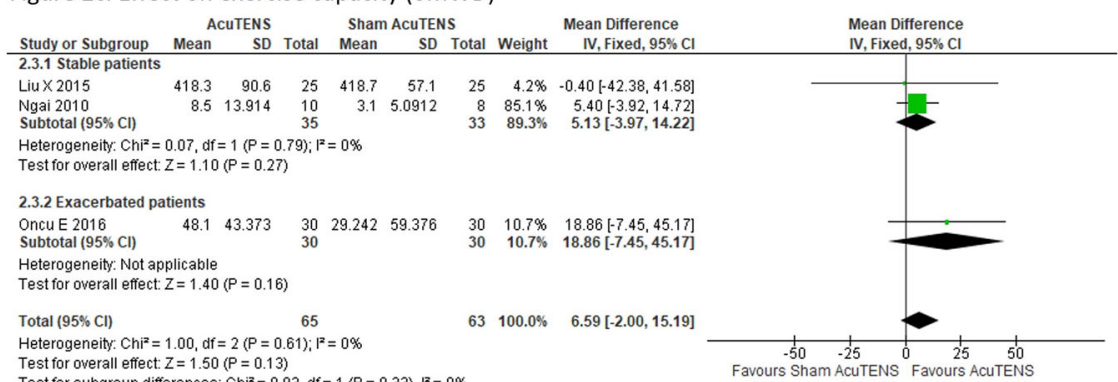

Test for subgroup differences: $C \mathrm{Ch}^{2}=0.93, \mathrm{df}=1(P=0.33), \mathrm{I}^{2}=0 \%$

Figure 2d. Effect on FEV

\begin{tabular}{|c|c|c|c|c|c|c|c|c|}
\hline \multirow{3}{*}{ Study or Subgroup } & \multicolumn{3}{|c|}{ ACUTENS } & \multicolumn{3}{|c|}{ Sham AcuTENS } & \multirow{2}{*}{$\begin{array}{l}\text { Std. Mean Difference } \\
\text { IV, Random, } 95 \% \mathrm{Cl}\end{array}$} & \multirow{2}{*}{$\begin{array}{l}\text { Std. Mean Difference } \\
\text { IV, Random, } 95 \% \mathrm{Cl}\end{array}$} \\
\hline & Mean & SD & Total & Mean & SD & Total & & \\
\hline & \multicolumn{8}{|c|}{ 2.4.1 Stable patients } \\
\hline Lau 2008 & 0.13 & 0.09 & 23 & 0.01 & 0.03 & 23 & $1.76[1.07,2.45]$ & 十 \\
\hline Liu $\times 2015$ & 53.6 & 19.5 & 25 & 39 & 16.8 & 25 & $0.79[0.21,1.37]$ & $\rightarrow$ \\
\hline Ngai 2010 & 0.86 & 0.4111 & 10 & 0.75 & 0.2828 & 8 & $0.29[-0.65,1.23]$ & + \\
\hline Ngai 2011 & 0.66 & 0.187 & 22 & 0.62 & 0.148 & 22 & $0.23[-0.36,0.83]$ & + \\
\hline Shou YQ 2014 & 1.77 & 0.5 & 15 & 1.68 & 0.46 & 15 & $0.18[-0.54,0.90]$ & $t$ \\
\hline Wen Q 2011 & 20.6 & 12.6 & 20 & 19.6 & 10.3 & 20 & $0.09[-0.53,0.71]$ & - \\
\hline \multicolumn{9}{|c|}{ 2.4.2 Exacerbated patients } \\
\hline Oncu E 2016 & 1.31 & 0.71 & 30 & 1.36 & 0.56 & 30 & $-0.08[-0.58,0.43]$ & - \\
\hline
\end{tabular}

Figure 2e. Effect on FVC

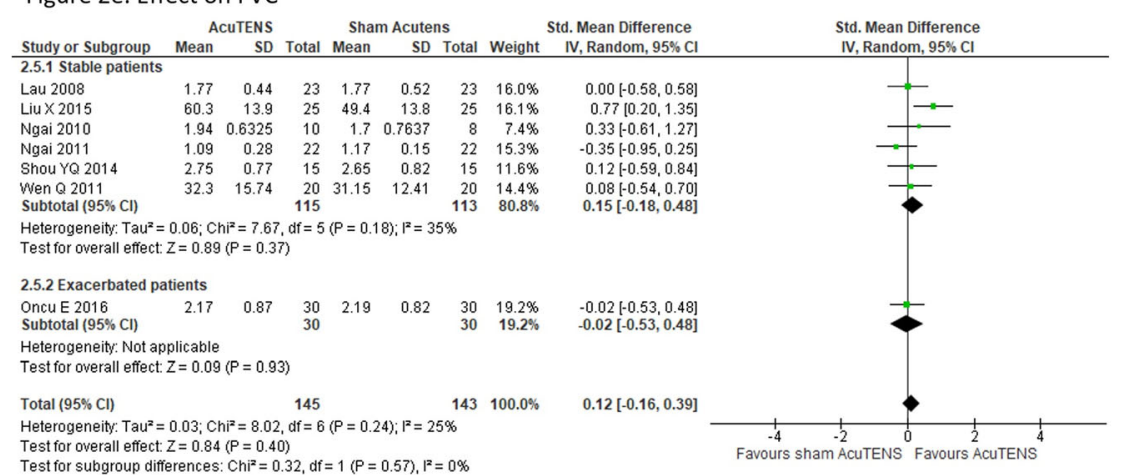

Fig. 2 Meta-analysis of AcuTENS vs Sham 
scale in the moxibustion group compared with the control after a 12 week intervention (MD: $-1.70, \mathrm{Sd}: 0.47$ vs MD: -1.03 , Sd: 0.18, $p<0.05)$.

Quality of life Five trials assessed QoL, four using the SGRQ [8, 20, 24, 26] and one using the CAT [25]. One trial did not report total scores of the SGRQ but only scores from each component separately [20], we calculated total scores using that data. Meta-analysis was not possible due the great heterogeneity $\left(\mathrm{I}^{2}=92 \%\right)$. Out of five trials, four showed an improvement in QoL in the moxibustion group $[8,20,24,25]$ while one trial did not observe statistical differences [26]. (Fig. 3a).
Exercise capacity Only one trial studied the effect of moxibustion on exercise capacity [20]. In this trial with 100 participants the authors reported an improvement in this outcome using the 6MWD $(\mathrm{t}=3.568, p<0.001)$, but no difference between groups was reported.

Lung function Ten trials analysed lung function, all using $\mathrm{FEV}_{1}[16-18,20-24,26,27]$, eight using $\mathrm{FEV}_{1} /$ FVC [16, 17, 20-24, 27] and three trials using FVC $[18,20,21]$. Meta-analysis was not possible due to the high heterogeneity for $\mathrm{FEV}_{1}$ and $\mathrm{FEV}_{1} / \mathrm{FVC}\left(\mathrm{I}^{2}=93\right.$ and $80 \%$ respectively). Seven trials for $\mathrm{FEV}_{1}$ showed a statistical benefit for moxibustion [16, 17, 20, 22-24, 27] and

Figure 3.a Quality of Life: SGRQ

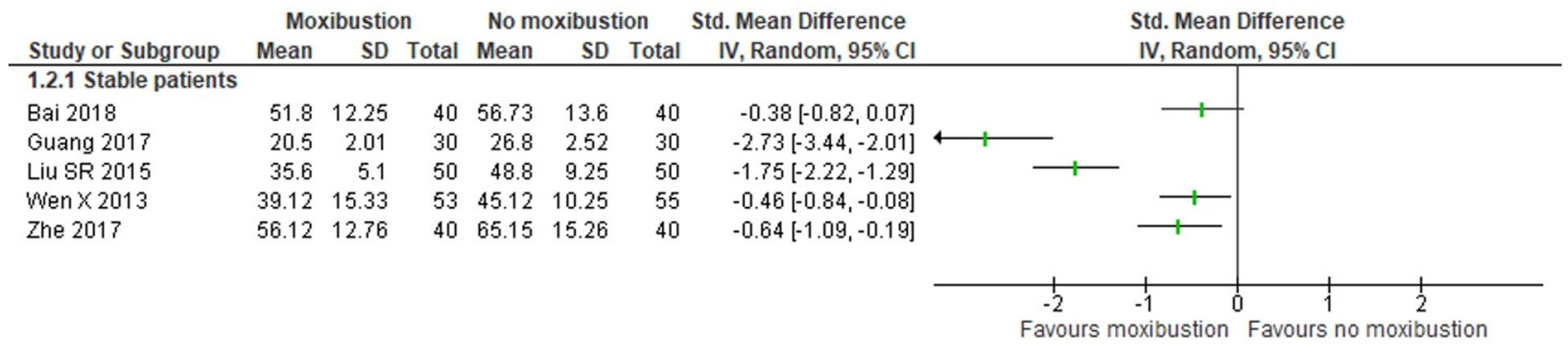

Figure 3.b FEV 1

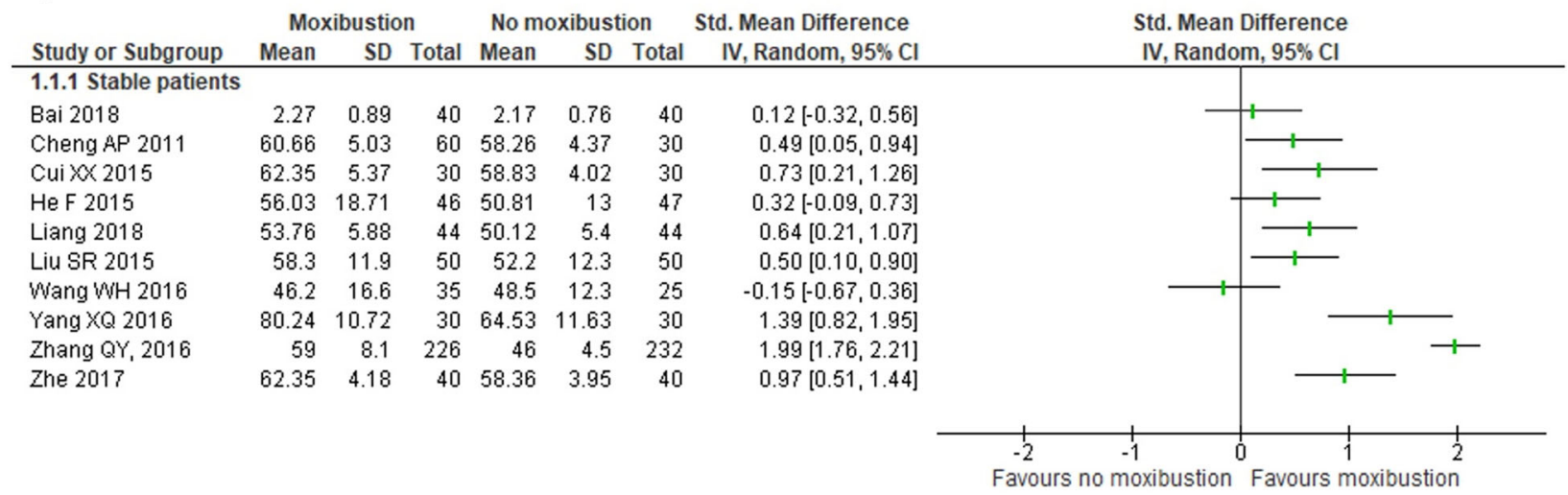

Figure 3.c FEV $1 / F V C$

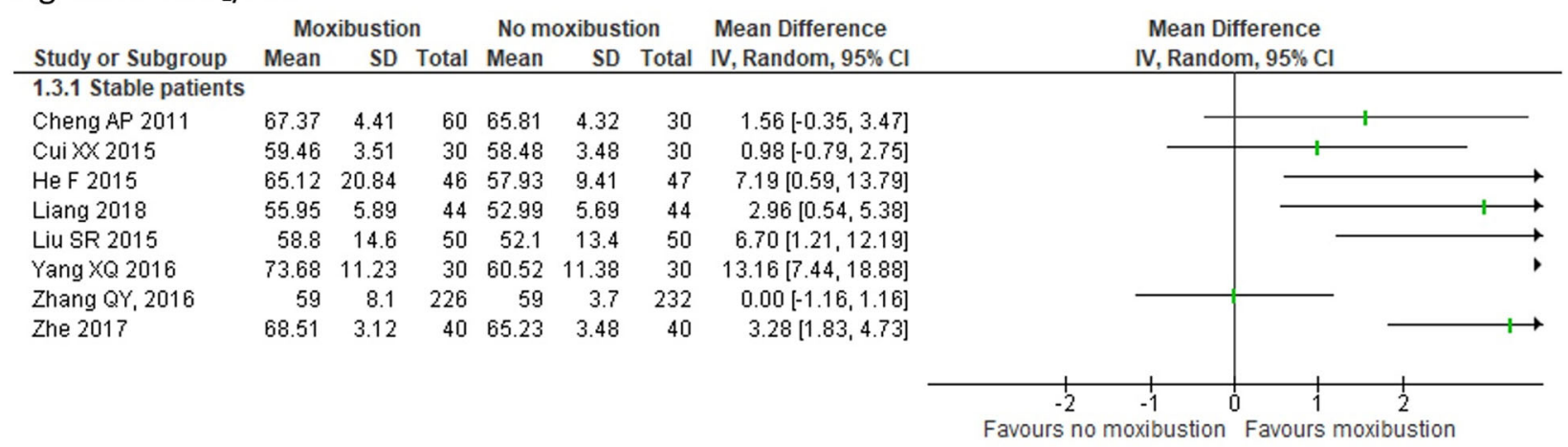

Fig. 3 Meta-analysis of Moxibustion vs no Moxibustion 
three showed no effect [18, 21, 26] (Fig. 3b). Five trials for $\mathrm{FEV}_{1} / \mathrm{FVC}$ showed benefits for moxibustion [17, 20, 21, $24,27]$ and the other three did not show statistical differences [16, 22, 23] (Fig. 3c).

Adverse events No trials reported adverse events.

\section{Acupressure technique}

\section{Acupressure vs. sham acupressure}

Dyspnoea Dyspnoea was assessed in 2 trials (3 publications) using VAS (2 trials) [29, 30], the modified Borg scale (1 trial) [25] and the Pulmonary Function Status and Dyspnoea Questionnaire-Modified (PFSDQ-M) (1 trial) [31]. Meta-analysis was not possible since the study by Maa [30] used a cross-over design. Wu et al. reported a statistical improvement in the real acupressure group using VAS (43.43 $\mathrm{mm}$ vs $48.97 \mathrm{~mm} p<0.01)$ [29] and the dyspnoea subscale of the PFSDQ-M (MD: -0.98 , $p<0.01$ ) [31]. Maa [30] reported a statistical improvement in the real acupressure group using the VAS $(p=0.009)$ but not with the Borg scale $(p=0.38)$.

Exercise capacity Two trials, both including stable patients, studied the effect of acupressure on exercise capacity using the 6MWD [30,31]. Again, due to the cross over design used by Maa [30], a meta-analysis was not performed. While Maa did not report statistical differences between groups $(p=0.67)$, Wu et al. [26] did report a statistical improvement in the acupressure group $(p<0.001)$, none of the trials reported mean differences between groups.

Anxiety Two trials reported anxiety in stable patients $[30,31]$. The scales used for measuring were the Spielberger's State Anxiety Inventory (SSAI) [31] and the anxiety subscale of the Bronchitis-Emphysema Symptom Checklist (BESC) [30]. Meta-analysis was not possible due to the cross-over design of Maa et al. Wu et al. reported statistical improvement in the acupressure group compared with sham intervention in the SSAI (-8.50 vs $-0.14, \mathrm{p}<0.01)$ [31]. Ma et al. reported a significant improvement in the acupressure group using the BESC $(p<0.05)$ but the mean difference between groups was not reported [30].

Depression One trial [29], which included stable participants, reported a greater reduction on the Geriatric Depression Scale (GDS) in the acupressure group than the sham group $(-2.09$ vs $0.14 \mathrm{p}<0.001)$.

\section{Acupressure vs no acupressure}

Dyspnoea Only one trial, comparing the addition of acupressure to usual care with usual care alone in patients under mechanical ventilation, assessed dyspnoea
[28]. The authors reported a statistical reduction in dyspnoea of 6.77 points on VAS scale (SE: 2.59, $p=0.009$ ).

Quality of life Three trials studied QoL in stable participants, with one trial using the SGRQ [32], one trial using the CAT scale [33] and one trial using the Generic Quality of Life Inventory-74 (GQOL-74) [34]. The trial from $\mathrm{Wu}$ et al. [34] could not be included in the metaanalysis since authors did not report the GQOL-74 global score. Meta-analysis of the other two trials showed a statistically significant improvement on QoL (SMD: -0.63 95\%CI: - 0.88, - $0.39 \mathrm{I}^{2}=0 \%$ ) (Supplementary material 2.a).

Exercise capacity One trial assessed exercise capacity using the 6MWD in stable participants [33]. Authors reported a greater distance walked in the acupressure group compared with the control (MD: $384.38 \mathrm{~m}, \mathrm{SD}$ : 21.08 vs MD: $370.00 \mathrm{~m}, \mathrm{SD}: 23.74, p=0.010)$.

Anxiety Tree trials assessed anxiety, with two including stable participants [32, 35] and one with exacerbated participants [28]. The scales used were the VAS [28] and the Hamilton Anxiety Rating Scale (HAM-A) [32, 35]. Meta-analysis if the two trials with stable participants (332 participants) showed a statistical reduction of 4.83 points in the HAM-A scale $\left(95 \% \mathrm{CI}:-5.71,-3.94 \mathrm{I}^{2}=\right.$ $0 \%$ ) (Supplementary material 2.b). The trial including exacerbated participants did not report enough data to be included in the analysis but also reported a statistical improvement compared with the control group (VAS MD: -6.74, SE: 2.68, $p=0.011$ ).

Depression Only one trial including sable participants reported depression using the Hamilton Depression Rating Scale (HAM-D) [32]. In this trial authors reported lower depression levels in the experimental group after the intervention (MD: 12.4, SD:4.36 vs MD: 19.1, SD: $6.1, p<0.05)$.

Lung function Lung function was assessed in two trials with stable participants [32, 33], both trials reported no statistical differences between acupressure and control group in $\mathrm{FEV}_{1}, \mathrm{FVC}$ and $\mathrm{FV}_{1} / \mathrm{FVC}$.

Adverse events Only one trial considered the possibility of adverse events and reported no skin reactions at the areas where acupressure was applied [30].

\section{Ear acupunture}

Ear acupuncture vs sham ear acupuncture

Only one trial, including 30 exacerbated participants, compared ear acupuncture vs sham [36]. In this trial the investigators found a significant improvement in quality 
of life and lung function $\left(\mathrm{FEV}_{1}, \mathrm{FEV}_{1} / \mathrm{FVC}\right)(p<0.05)$ after a 20-day intervention. Data on the effect size was not reported.

\section{Ear acupuncture vs no ear acupuncture}

Dyspnoea Only one trial including stable participants assessed dyspnoea, reporting a statistical reduction in the mMRC scale in the two ear acupuncture groups compared with the control [40].

Lung function Four trials assessed lung function [37-40], all of them assessing $\mathrm{FEV}_{1}$ and $\mathrm{FEV}_{1} / \mathrm{FVC}$. Meta-analysis (224 participants) showed no statistical difference for this comparison on $\mathrm{FEV}_{1}$ (MD: 0.05 L 95\%CI: - 0.05, 0.14 I ${ }^{2}=$ 0\%) (Supplementary material 3.a) and $\mathrm{FEV}_{1} / \mathrm{FVC}$ (MD: 1.03 95\%CI: - 1.16, $3.22 \mathrm{I}^{2}=0 \%$ ) (Supplementary material 3.b).

\section{Ear acupuncture vs drugs}

One trial compared a single intervention treatment with salbutamol inhalation in 32 exacerbated patients [41]. The authors found a significant improvement in $\mathrm{FEV}_{1}$ $(\mathrm{t}=2.62, \mathrm{p}<0.05)$, and no difference in $\mathrm{FVC}(\mathrm{t}=0.34$, $p>0.05)$.

Adverse events No trials reported adverse events.

\section{Acupressure plus ear acupuncture}

One trial analysed the effect of acupressure combined with ear acupuncture plus standard therapy compared with standard therapy alone in lung function [42]. This trial, which included 120 stable participants, reported a significant improvement in the intervention group in $\mathrm{FEV}_{1}, \mathrm{FVC}, \mathrm{FEV}_{1} / \mathrm{FVC}$ compared with the control group $(p<0.05)$.

\section{Cupping}

Only one trial with 60 participants studied the effect of cupping [43]. The authors reported an improvement in cough, expectoration, dyspnoea and wheezing $(\mathrm{p}<0.05)$ using the TCM syndrome integral. Data on the effect size was not reported. No adverse events were reported.

\section{Discussion}

This is the first systematic review that evaluates independently the effectiveness of the different non-filiform needle acupuncture techniques for COPD. For practical reasons we only analysed the techniques that are most commonly used outside China; AcuTENS, moxibustion, acupressure, ear acupuncture and cupping (33 trials).

Our results do not show strong evidence for any nonfiliform acupuncture techniques, only acupressure seams to improve dyspnoea and anxiety, based in low quality trials. The low number of trials assessing important outcomes, the great heterogeneity and the small size of most of the studies implies that these results must be interpreted with caution.

Overall, only six meta-analysis could be performed, with only two of them showing positive results. Heterogeneity was a big issue, even using subgroups for stable and exacerbated participants. This issue was even greater for the moxibustion technique, with no possible metaanalysis from three comparisons. We also found that very few trials reported important clinical outcomes commonly assessed in COPD such as dyspnoea (7/33), QoL (11/33), exercise capacity (7/33), anxiety (5/33) and depression (2/33). No important adverse events were reported for any technique.

Dyspnoea was improved in all 3 acupressure trials (2 trials vs sham and 1 trial vs no acupressure). For the other non-filiform acupuncture techniques only 1 trial using ear acupuncture and 2 trials using AcuTENS had positive results on dyspnoea, remarkably, both studies used a single session treatment. Acupuncture techniques mainly target the stimulation of cutaneous and muscular afferent fibres which lead to the stimulation of many brain nuclei networks, leading to the release of opioid peptides [44]. This mechanism has been usually accepted to explain acupuncture analgesic effects, but could also be used to explain acupuncture effects on dyspnoea, since endogenous opioids modulate dyspnoea in patients with COPD [45]. This mechanism has been better studied for the AcuTENS technique, which has been shown to increase B-endorphin levels which correlate with respiratory rate reduction [12]. However, improvements on dyspnoea were only seen for AcuTENS in single session trials but not in longer trials.

Quality of life is one of the main patient-related outcomes in clinical trials, however it was only studied in 11 trials. While meta-analysis of the 3 AcuTENS trials showed a tendency for improvement, results were not statistically significant. Quality of life was improved in 5 moxibustion trials with stable participants ( 2 vs sham and 3 vs no acupressure) and one ear acupuncture trial with exacerbated participants, all low-quality trials. It is to note that bowth trials comparing acupressure with sham acupressure lasted from 14 to 20 days and while seeing those changes in exacerbated patients in such a short time seems reasonable, it is quite surprising to find them in stable participants.

Exercise capacity is an important marker that results from a range of effects of COPD. Statistical differences in the 6MWD were not seen in the meta-analysis of the 3 AcuTENS trials however, while in the two trials with stable participates differences between groups were inexistent, in the trial with exacerbated participants an improvement was observed although CI was too wide to show statistical significance. One moxibustion trial and two acupressure trials reported benefits while another 
acupressure trial did not, but only one did report data of differences between groups.

Anxiety is strongly related to dyspnoea perception in COPD since the lack of breath is one of the most limiting symptoms experimented by patients. This correlation is seen in acupressure trials were all trials showing improvement on anxiety ( 5 trials) and dyspnoea ( 3 trials). Surprisingly this outcome was not studied in any other technique.

Finally, the effects on pulmonary function should be taken with caution. The heterogeneity of the results and the unexpected changes because the chronicity of the disease, prevent to any deeply interpretation. This outcome has been the most studied in the included trials (25/33), however, since pulmonary function is not expected to improve in COPD, no matter what treatment is used, we think future trials should not consider it as one of the main outcomes.

Coyle et al. [46] and Wang et al. [37] previously examined the effect of different acupuncture techniques for COPD. Coyle reviewed 16 trials (published between 1995 and 2007) of all kinds of acupuncture interventions, including non-pharmacological (filiform needle, moxibustion and acupressure, etc.) and pharmacological (herb plasters on acupuncture points). They concluded that acupuncture was beneficial for COPD patients in outcomes like dyspnoea, exercise capacity and quality of life, however evidence was low due to the methodological flaws of the included studies. Moreover, they plotted together all acupuncture techniques which might have caused some bias in the results. Wang's review included 19 trials and concluded that acupuncture might be effective in improving functional effects and quality of life in COPD patients. However, this review only included acupuncture techniques such as manual acupuncture, warm acupuncture, electroacupuncture and ear acupuncture, but all other non-invasive technique such as single moxibustion, acupressure or acuTENS were excluded.

This review has several limitations. First, due to the great heterogeneity between trials, only seven metaanalyses could be performed. Secondly, important clinical outcomes for COPD, such as dyspnoea, QoL, exercise capacity, anxiety and depression were only studied in a small number of trials, reducing the number of trials that could be combined in each meta-analysis. Combined with the first point, this led to a low inspection efficiency of the results. Third, the trials included had inadequate reporting, especially for random sequence generation and allocation concealment, meaning that they had an uncertain risk of bias. Fourth, although no important adverse events were reported, this outcome has not been systematically explored. Fifth, it is difficult to extrapolate these results for different populations since, except one, all trials were performed in China. Finally, we identified other non-pharmacological acupuncture interventions in this review, such as catgut implant, thick needle, acupoint incision, wet cupping, floating needle and intradermal needle, however, due to the rare use of those techniques outside China, and the complexity of the review, we did not include them in this review analysis.

\section{Conclusions}

No strong evidence was found for any of the included outcomes for patients with COPD treated with nonfiliform needle acupuncture techniques. Acupressure could improve dyspnoea, quality of life and anxiety, but this is only based on low quality trials.

Evidence is very low in this review due to the unclear risk of bias in the trials included, and the great heterogeneity between them. Further studies should include main outcomes for COPD assessment such as dyspnoea, quality of life, exercise capacity and anxiety since we found many studies mainly targeting pulmonary function. Well-designed trials are needed to elucidate its possible role in the treatment of COPD.

\section{Supplementary information}

Supplementary information accompanies this paper at https://doi.org/10. 1186/s12906-020-02899-3.

Additional file 1. Supplementary material 1: Search strategies.

Additional file 2. Supplementary material 2: Meta-analysis of Acupressure vs no Acupressure.

Additional file 3. Supplementary material 3: Meta-analysis of Ear acupuncture vs no Ear acupuncture.

\section{Acknowledgments}

The reviewers would like to thank Alice Jones and Shirley Ngai from the study of Ngai et al. [11], and Emine Öncü from the Öncü E et al. [13] study, for corresponding with them in their attempts to gain additional data/ information.

We would also like to thank Ivan Solà and Marta Roqué from the Iberoamerican Cochrane Centre for helping in the database search and with technical and logistic support for the review, Maria Victoria Leo for the translation and editing of the manuscript, and Si Wen Chen for translating Chinese references. The corresponding author is a Phd candidate at the Universitat Autònoma de Barcelona, Spain.

\section{Authors' contributions}

CF has been responsible for writing the protocol of the study, performing the electronic search in English, the extraction and analysis of the data and the writing and the submission of the manuscript. JV and MSR made substantial contributions to the conception and design of the review, extraction, analysis and interpretation of the data and writing and reviewing the manuscript. YF, and CW and $J \mathrm{~L}$ are responsible for the Chinese language electronic search, and data abstraction and contributed to the writing and reviewing of the manuscript. $\mathrm{NH}, \mathrm{RX}, \mathrm{XT}, \mathrm{RH}, \mathrm{NG}$ and MSM, are responsible for trials inclusion, data extraction and critically reviewing the final manuscript. MY and LW are responsible for the latest update of Chinese databases including, trail inclusion and data extraction process. All authors read and approved the final manuscript. 


\section{Funding}

This work was supported by a grant from the Professional College of Physiotherapists of Catalonia.

\section{Availability of data and materials}

All data generated or analysed during this study are included in this published article and its supplementary information files.

During the preparation of this paper Carles Fernández was also given a grant from the Spanish Education Ministry.

\section{Ethics approval and consent to participate}

Not applicable.

\section{Consent for publication}

Not applicable.

\section{Competing interests}

The authors declare that they have no competing interests.

\section{Author details}

${ }^{1}$ School of Health Science Blanquerna, Ramon Llull University, Barcelona, Spain. ${ }^{2}$ Global Research on Wellbeing (GRoW) Research Group, Ramon Llull University, Padilla 326-332, 08025 Barcelona, Spain. ${ }^{3}$ Centre for Evidence-Based Chinese Medicine, Beijing University of Chinese Medicine, Beijing, China. ${ }^{4}$ Faculty of Health Science and Welfare, University of Vic, Vic, Spain. ${ }^{5}$ Residència Jaume Batlle, Pere Mata Foundation, Barcelona, Spain.

\section{Received: 5 January 2019 Accepted: 18 March 2020}

\section{Published online: 06 May 2020}

\section{References}

1. GBD 2015 Chronic Respiratory Disease Collaborators. Global, regional, and national deaths, prevalence, disability-adjusted life years, and years lived with disability for chronic obstructive pulmonary disease and asthma, 19902015: a systematic analysis for the Global Burden of Disease Study 2015. Lancet Respir Med. 2017;(9):691-706.

2. López-Campos JL, Tan W, Soriano JB. Global burden of COPD. Respirology. 2016;21(1):14-23.

3. Vogelmeier CF, Criner GJ, Martínez FJ, Anzueto A, Barnes PJ, Bourbeau J, Celli BR, Chen R, Decramer M, Fabbri LM, Frith P, Halpin DMG, Varela MVL, Nishimura M, Roche N, Rodríguez-Roisin R, Sin DD, Singh D, Stockley R, Vestbo J, Wedzicha JA, Agustí A. Global strategy for the diagnosis, management, and prevention of chronic obstructive lung disease 2017 report. GOLD executive summary. Am J Respir Crit Care Med. 2017;195(5): 557-82

4. Miravitlles M, Soler-Cataluña JJ, Calle M, Molina J, Almagro P, Quintano JA, Riesco JA, Trigueros JA, Piñera P, Simón A, Rodríguiez-Hermosa JL, Marco E, López D, Coll R, Coll-Fernández R, Lobo MA, Díez J, Soriano JB, Ancochea J. Spanish guideline for COPD (GesEPOC). Update 2014. Arch Bronconeumol. 2014;50(Suppl 1):1-16.

5. Higgins JPT, Green S. Cochrane Handbook for Systematic Reviews of Interventions Version 5.1.0 [updated March 2011]. Cochrane Collaboration. 2011; Available from: www.handbook.cochrane.org. [cited 2018 Mar 26].

6. Moher D, Shamseer L, Clarke M, Ghersi D, Liberati A, Petticrew M, et al. Preferred reporting items for systematic review and meta-analysis protocols (PRISMA-P) 2015 statement. Syst Rev. 2015:4:1.

7. Higgins JPT, Altman DG, Gotzsche PC, Juni P, Moher D, Oxman AD, et al. The Cochrane Collaboration's tool for assessing risk of bias in randomised trials. BMJ. 2011;343(2):d5928.

8. Wen X, Chen CY, Liang MA. Clinical research of the Moxa cone Moxibustion for improving life quality of chronic obstructive pulmonary disease (COPD) patients in the tranquilization period. World Chinese Med. 2013;8(06):658-60.

9. Lau KSL, Jones AYM. A single session of Acu-TENS increases FEV1 and reduces dyspnoea in patients with chronic obstructive pulmonary disease: a randomised, placebo-controlled trial. Aust J Physiother. 2008;54(3):179-84.

10. Liu X, Fan T, Lan Y, Dong S, Fu J, Mao B. Effects of transcutaneous electrical Acupoint stimulation on patients with stable chronic obstructive pulmonary disease: a prospective, single-blind, randomized, placebo-controlled study. J Altern Complement Med N Y N. 2015;21(10):610-6.

11. Ngai SPC, Jones AYM, Hui-Chan CWY, Ko FWS, Hui DSC. Effect of 4 weeks of AcU-TENS on functional capacity and beta-endorphin level in subjects with chronic obstructive pulmonary disease: a randomized controlled trial. Respir Physiol Neurobiol. 2010;173(1):29-36.

12. Jones AYM, Ngai SPC, Hui-Chan CWY, Yu HPM. Acute effects of Acu-TENS on FEV1 and blood B-endorphin level in chronic obstructive pulmonary disease. Altern Ther Health Med. 2011;17(5):8-13.

13. Öncü $\mathrm{E}$, Zincir $\mathrm{H}$. The effect of transcutaneous electrical nerve stimulation in patients with acute exacerbation of chronic obstructive pulmonary disease: randomised controlled trial. J Clin Nurs. 2017;26(13-14):1834-44.

14. Shou YQ, Ge HQ, Dai ZN, et al. Effects of percutaneous electrical nerve stimulation in Feishu acupoint on lung function and induced tumor necrosis factor-a concentration in patients with chronic obstructive pulmonary disease. Chinese J Phys Med Rehabil. 2014;9:705-8.

15. Wen Q, Li N, Yu P-M. Randomized controlled trial on the effect of transcutaneous electrical nerve stimulation at Dingchuan (EX-B1) on the pulmonary function of patients with COPD at acute stage. Zhongguo Zhen Jiu Chin Acupunct Moxibustion. 2011;31(2):97-100.

16. Zhang QY, LV XD, Feng Y. Long-term effect of Chinese medicine moxibustion on COPD combined with type 2 respiratory failure. Chinese $J$ Tradit Med Sci Technol. 2016;23(02):193-5.

17. Yang $X Q$, Fu DY. Effects of moxibustion on chronic obstructive pulmonary disease with pulmonary qi deficiency. People's Military Med Officer. 2016; 59(10):1036-7.

18. Wang WH, Qiu YZ, Huang JM, Chen MJ. Efficacy observation and nursing of moxibustion during stable period of chronic obstructive pulmonary disease. Nei Mongol J Tradit Chinese Med. 2016;35(03):69-70.

19. Tang J. Efficacy observation and nursing of moxibustion for treatment of chronic obstructive pulmonary disease in the elderly. J Beneficial Readines Drug Informations Med Advices. 2012;10(04):456.

20. Liu SR. Clinical efficacy of Chinese medicine moxibustion on stable chronic obstructive pulmonary disease. Pract J Cardiac Cereb Pneumal Vasc Dis. 2015;23(4):150-2.

21. He F, He SF, Mai JY. Combination of ginger moxibustion on Feishu (BL13) and compound Methoxyphenamine for chronic obstructive pulmonary disease at remission stage: a report of 46 cases. Shanghai J Tradit Chinese Med. 2013;47(06):54-5.

22. Cui XX, Liang GL. Treating 60 cases of chronic obstructive pulmonary disease in TCM. Clin J Chinese Med. 2015;7(25):10-2.

23. Cheng AP, Shu CX. The investigation the heat sensitive Point's Moxibustion effect of treating chronic obstructive pulmonary disease (COPD). Chinese J Tradit Chinese Med Pharmacy. 2011;29(06):1355-7.

24. Zhe $Y$, Yani Xue $Y, N i X Q$. Effect of heat-sensitive moxibustion on pulmonary function and quality of life in patients with stable COPD. J Clin Med Pract. 2017:21(08):42-5.

25. Guang T, Gao PF, Chen B, Li Z, Jiang M. Clinical study on treatment of chronic obstructive pulmonary disease in stable phase with six-hole moxibustion moxibustion. Shanghai J Acupunct Moxibustion. 2017;36(07):799-802.

26. Bai L, Dai S, Lin Q. Nursing study on lung function and quality of life of patients with stable COPD during traditional Chinese medicine moxibustion. J Clin Nurs Pract. 2018;3(09):8-9.

27. Liang YY. Clinical observation on 44 cases of stable period of chronic obstructive pulmonary disease treated with Acupoint Thermosensitive suspension Moxibustion. J Tradit Chin Med. 2018;59(09):765-8.

28. Tsay SL, Wang JC, Lin KC, Chung UL. Effects of acupressure therapy for patients having prolonged mechanical ventilation support. J Adv Nurs. 2005:52(2):142-50.

29. Wu H-S, Lin L-C, Wu S-C, Lin J-G. The psychologic consequences of chronic dyspnea in chronic pulmonary obstruction disease: the effects of acupressure on depression. J Altern Complement Med. 2007;13(2): 253-61.

30. Maa SH, Gauthier D, Turner M. Acupressure as an adjunct to a pulmonary rehabilitation program. J Cardpulm Rehabil. 1997;17(4):268-76.

31. Wu H-S, Wu S-C, Lin J-G, Lin L-C. Effectiveness of acupressure in improving dyspnoea in chronic obstructive pulmonary disease. J Adv Nurs. 2004;45(3): 252-9.

32. Guo $X$, Zhan $X P$, Jin $X Z$, Huang QH, Jin H, Hu L, Yu NM, Qi X. Effect of acupressure combined with respiratory function exercise on pulmonary function and quality of life in patients with chronic obstructive pulmonary disease at stable stage. Chinese Gen Pract. 2017;20(S2):345-7.

33. Huang YL, Feng LY, Danyu L. Clinical observation on acupressure for pulmonary rehabilitation in patients with chronic pulmonary Qi deficiency and COPD. Med Front. 2018;8(3):344-5. 
34. Wu H, Zhang JW. Effect of acupressure on quality of life in elderly patients with chronic obstructive pulmonary disease. Nurs Pract Res. 2017;14(11): $132-3$.

35. Xu Y. Effect of Acupressure of Traditional Chinese Medicine on Anxiety of Patients with Chronic Obstructive Pulmonary Disease. J Liaoning Univ Tradit Chinese Med. 2018;20(02):127-9.

36. Cao L, Shao MM, Liu ZY. Study on the quality of life of patients with acute exacerbation of COPD treated by acupuncture and auricular points. Yunnan J Tradit Chinese Med. 2012;33(4):53-5.

37. Jin RF, Lu YQ, Liu G. A clinical study on ear Acupoint application therapy of chronic obstructive pulmonary diseases. Chin Arch Tradit Chin Med. 2009; 27(6):1283-4.

38. Pang CL, Li ZC. Impact of auricular pressure therapy on malnutrition patients with COPD. China Modern Doctor. 2016;54(12):142-4.

39. Pang $C L, L i Z C$. Effects of invigorating kidney and spleen method on nutritional status of COPD in spleen and kidney deficiency type. Guangming J Chinese Med. 2014;29(09):1863-4.

40. Li D, Hong X, Hang R. Effect of ear acupuncture on the rehabilitation of patients with chronic obstructive pulmonary disease. Nurs J Chinese Peoples Liberation Army. 2017;34(08):31-34+47.

41. Hu ZH, Cai H. The effect of auricular acupuncture to improve the airway function of COPD patients. J Clin Acupunct Moxibustion. 1997:13(11):22-3.

42. Rao JF, Xie Y. Effect of acupressure on the head and ear acupuncture on the sleep quality of patients with insomnia in COPD. Guangming J Chinese Med. 2017;32(22):3289-91.

43. Xiao W, Wang Y, Kong HB, et al. Clinical observation of cupping of BeiShu point to stable COPD. Clin J Tradit Chinese Med. 2009;21(5):420-1.

44. Zhao Z-Q. Neural mechanism underlying acupuncture analgesia. Prog Neurobiol. 2008;85(4):355-75.

45. Mahler DA, O'Donnell DE. Recent advances in dyspnea. Chest. 2015;147(1): 232-41.

46. Coyle ME, Shergis JL, Huang ET-Y, Guo X, Di YM, Zhang A, et al. Acupuncture therapies for chronic obstructive pulmonary disease: a systematic review of randomized, controlled trials. Altern Ther Health Med. 2014;20(6):10-23.

\section{Publisher's Note}

Springer Nature remains neutral with regard to jurisdictional claims in published maps and institutional affiliations.

Ready to submit your research? Choose BMC and benefit from:

- fast, convenient online submission

- thorough peer review by experienced researchers in your field

- rapid publication on acceptance

- support for research data, including large and complex data types

- gold Open Access which fosters wider collaboration and increased citations

- maximum visibility for your research: over $100 \mathrm{M}$ website views per year

At $\mathrm{BMC}$, research is always in progress.

Learn more biomedcentral.com/submissions 